\title{
CORRIGENDUM
}

\section{Ovarian shielding allows ovarian recovery and normal birth in female hematopoietic SCT recipients undergoing TBI}

K Nakagawa, Y Kanda, H Yamashita, S Nakagawa, N Sasano, K Ohtomo, K Oshima, K Kumano, N Ban, Y Nannya, M Kurokawa and S Chiba

Bone Marrow Transplantation (2008) 42, 839; doi:10.1038/bmt.2008.393

Correction to: Bone Marrow Transplantation (2008) 42, 697-699; doi:10.1038/bmt.2008.234

Since the publication of this article, the authors have identified that the name Y Minamitani was incorrect. The correct name is Y Nannya and the full authors list is shown above. The authors would like to apologize for any inconvenience this may have caused. 